\title{
e-Migrinter
}

9| 2012

Immigrés, illégaux, réfugiés. Questions sur les enquêtes et les catégories

\section{Les non-dits de l'asile en Afrique subsaharienne} approche par une enquête à passages répétés dans un site de réfugiés en Zambie (1993-2007)

\section{Véronique Lassailly-Jacob}

\section{OpenEdition}

\section{Journals}

Édition électronique

URL : https://journals.openedition.org/e-migrinter/763

DOI : 10.4000/e-migrinter.763

ISSN : 1961-9685

\section{Éditeur}

UMR 7301 - Migrinter

Édition imprimée

Date de publication : 20 juillet 2012

Pagination : 29-46

ISSN : 1961-9685

\section{Référence électronique}

Véronique Lassailly-Jacob, «Les non-dits de l'asile en Afrique subsaharienne », e-Migrinter [En ligne], 9 | 2012, mis en ligne le, consulté le 20 mai 2021. URL : http://journals.openedition.org/e-migrinter/763 DOI : https://doi.org/10.4000/e-migrinter.763 


\section{Les non-dits de l'asile en Afrique subsaharienne : approche par une enquête à passages répétés dans un site de réfugiés en Zambie (1993-2007)}

Véronique Lassailly-Jacob

A d'indépendance et dans un contexte de guerre froide, de nombreux pays d'Afrique subsaharienne, comme l'Angola ou le Mozambique, ont connu des guerres civiles interminables qui produisirent des milliers de réfugiés et de personnes déplacées. Les premiers trouvèrent refuge dans les pays limitrophes où ils furent regroupés, assistés et protégés par le Haut commissariat aux Réfugiés de l'ONU (HCR) et de nombreuses Organisations Non Gouvernementales (ONG) dans des camps ou des sites agricoles. Cet article traite des conditions de la recherche en sciences sociales dans les camps de réfugiés et des enseignements que l'on peut en tirer sur les non-dits de l'asile en Afrique subsaharienne.
Introduction

En Afrique, pendant trente ans, de 1960 à 1990, le HCR privilégia la solution de "l'aide à l'installation sur place» dans les pays de premier accueil pour assister et protéger des réfugiés de longue durée, en nombre, et en majorité d'origine rurale. Cette solution est décrite en ces termes par Yves Beigbeder, juriste et ancien fonctionnaire onusien : "dans la mesure du possible, l'aide à l'installation sur place prend la forme de projets visant à promouvoir l'autonomie des réfugiés sur le plan socioéconomique et leur intégration dans les pays d'asile, permettant ainsi au HCR de mettre fin progressivement à ses activités de soin et d'entretien » (Beigbeder, 1999 : 77). En effet, cette solution s'est traduite en milieu rural par des projets d'assistance agricole dispensés à des réfugiés ruraux qui étaient soit dispersés dans le milieu local (cas de la Guinée et de la Côte d'Ivoire), soit regroupés dans des sites agricoles ${ }^{1}$ (Soudan, Tanzanie, Ouganda, RDC, Zambie) qui avaient pour particularité de mettre à disposition des réfugiés des terres arables à cultiver. L'objectif était de créer des communautés économiquement viables et localement intégrées afin de les faire contribuer au développement du pays d'accueil et d'alléger ainsi le fardeau de la prise en charge des réfugiés à la fois pour les pays hôtes et pour la communauté internationale.

La Zambie a été l'un des pays d'Afrique australe avec la Tanzanie à adopter la solution de «l'aide à l'installation sur

${ }^{1}$ Ces sites agricoles sont dénommés en anglais refugee settlements et non pas refugee camp pour faire référence à l'installation durable des réfugiés. 
place ». Depuis son indépendance en 1964, le pays de Kenneth Kaunda, premier président zambien, a soutenu tour à tour les guérillas nationalistes des pays voisins, Angola, Mozambique, Namibie, Zimbabwe, offrant refuge à des milliers d'exilés. Par la suite, et aujourd'hui encore, la Zambie a ouvert ses frontières aux réfugiés de la République Démocratique du Congo (RDC), de la région des Grands Lacs (Rwandais, Burundais) et de l'Afrique de l'Est (Somaliens). La plupart de ces réfugiés reçurent un statut prima facie $^{2}$ et furent regroupés dans cinq sites agricoles gérés par le $\mathrm{HCR}^{3}$. Si la solution de "l'aide à l'installation sur place» a été longtemps encouragée en Zambie, notamment pour les réfugiés angolais, mozambicains et congolais, celle du rapatriement « librement consenti » joue depuis plusieurs années un rôle prépondérant. Et pour gérer l'asile, le gouvernement a créé en 1970 ses propres instruments juridiques en instituant un Commissariat aux réfugiés établi au sein du ministère de l'Intérieur. Ce Commissariat est chargé d'appliquer les clauses du Refugee Control Act qui stipule que tout réfugié ayant reçu un statut prima facie doit s'enregistrer dans un centre de transit, puis résider dans un camp ou un site agricole.

Pendant une quinzaine d'années, j'ai tenté de suivre la mise en application de la politique d'asile zambienne et son adéquation avec la solution durable de «l'aide à l'installation sur place». Pour cela, je suis retournée à plusieurs reprises en Zambie, entre 1992 et 2007, en choisissant de mener une enquête à passages répétés

${ }^{2}$ Le statut prima facie est une méthode collective de détermination du statut de réfugié. Dans ce cas, « chaque membre d'un groupe est considéré de prime abord comme réfugié, sur la base des circonstances objectives qui ont amené ses membres à fuir leur pays d'origine » (Begbeider, 1999 : 56)

${ }^{3}$ Dès 1965, la Zambie était signataire de toutes les grandes conventions relatives aux réfugiés, la Convention de 1951, celle du Protocole de 1967 ainsi que la Convention de l'OUA de 1969, et accueillait une forte délégation du HCR chargée de faire appliquer les trois solutions au problème des réfugiés, l'installation locale, le rapatriement et la réinstallation. auprès des différentes populations regroupées au cours de cette période dans un site agricole dénommé Ukwimi, situé au sud-est de la Zambie. Ce site avait été établi en 1987 pour accueillir environ 25000 réfugiés mozambicains fuyant la guerre civile (1976-1992) ${ }^{4}$ qui avait succédé à la guerre d'indépendance (1965-1975). Dans le site agricole d'Ukwimi qui s'étendait sur quelque $310 \mathrm{~km}^{2}$, les réfugiés mozambicains avaient été regroupés en 73 petits villages créés le long des pistes, à proximité des villages autochtones et chaque famille avait reçu une parcelle de 2 ha à cultiver.

L'objectif de cet article est d'interroger la faisabilité d'une méthodologie d'enquêtes dans un camp de réfugiés, l'enquête à passages répétés, et de mettre en lumière les éléments de compréhension d'une politique d'asile révélés par cette méthodologie. Construit en trois parties, cet article présente tout d'abord un bilan succinct des thèmes de recherche et des approches méthodologiques développés par des chercheurs en sciences sociales dans les camps et les sites de réfugiés. Il montre ensuite comment s'est élaborée la méthodologie d'enquêtes à passages répétés dans le site d'Ukwimi. Il dévoile enfin les apports et les limites de cette enquête en s'appuyant sur trois temps forts de la vie du site, l'urgence, le rapatriement et l'illégalité, trois périodes significatives des non-dits de la politique d'asile zambienne.

\section{Approches méthodologiques : les sciences sociales à l'épreuve des camps et des sites de réfugiés}

Les camps et les sites de réfugiés se sont multipliés en Afrique depuis les guerres d'indépendance des années 1960. Rappelons que ces camps, gérés par le HCR et par de nombreuses ONG, sont des lieux

${ }^{4}$ Cette guerre civile, à la fin de l'année 1992, avait causé la mort de plus d'un million de personnes, l'exil de quelque 1,7 million de réfugiés dans les pays limitrophes et un exode interne d'environ 3,5 millions de déplacés (US Committee for Refugees, 1993 : 67). 
d'assistance et de protection des réfugiés, mais aussi des lieux de cantonnement, de confinement de populations qui sont en quelque sorte mises à l'écart. Rappelons aussi que les sites agricoles représentent une forme particulière de regroupement promue par le HCR dans le cadre de sa politique d'aide à l'installation sur place. Ce sont de vastes espaces de terres arables mises à la disposition des réfugiés pour la pratique agricole. Si les objectifs de cette forme particulière d'installation sont d'apporter aux réfugiés, protection et assistance, ils se doublent d'une recherche d'auto-suffisance et d'intégration locale. Camps et sites agricoles, surveillés par l'armée et propriétés à la fois des États d'accueil, des organismes onusiens et des ONG, sont fermés au public et aux observateurs extérieurs, à l'exception des experts onusiens et des journalistes. Ce n'est que récemment que les chercheurs en sciences sociales ont pu y pénétrer. Depuis quand sont-ils entrés dans les camps, quels sont leurs objets de recherche et quelles méthodologies adoptent-ils?

\section{Les temps et les objets de la recherche dans les camps de réfugiés}

Comme le souligne Julie Baujard dans sa thèse sur les réfugiés à Delhi, le champ d'étude des réfugiés est récent et a d'abord été traité par «des juristes, des politologues, des spécialistes des relations internationales ou des économistes rattachés aux organisations internationales (onusiennes et humanitaires) » (Baujard, 2008 : 5). Aussi, la question des réfugiés a-t-elle été tout d'abord abordée selon une approche macro-sociale.

L'approche micro-sociale date des années 1980 lorsque, souligne Marion Fresia, «les déplacements de population s'intensifient dans les pays du Sud et que les réfugiés deviennent plus visibles» (Fresia, 2009 : 62). Barbara Harrell-Bond et Pierre Centlivres ont fait œuvre pionnière dans l'approche micro-sociale de la question des réfugiés et des camps. Barbara Harrell-Bond fonde en 1982 le premier centre de recherche sur les réfugiés, le Refugee Studies Programme à l'Université d'Oxford et crée les «Refugee studies». Anthropologue engagée, Barbara Harrell-Bond publie en 1986 «Imposing Aid. Emergency assistance to refugees" (Harrell-Bond, 1986), une première œuvre critique d'un programme humanitaire d'urgence «imposé » à des réfugiés, des Ougandais regroupés dans des camps au Sud Soudan ou dispersés dans la zone frontalière.

Pierre Centlivres fut également un anthropologue des camps de réfugiés, non par choix personnel mais par contrainte. Confronté aux crises successives que connue la société afghane depuis l'invasion soviétique en 1979, une société qu'il étudiait depuis de nombreuses années, il dût se résoudre - pour poursuivre ses recherches - à mener des enquêtes dans les camps de réfugiés afghans au Pakistan. Toutefois, il a toujours considéré ce nouveau terrain comme une épreuve, une crise pour l'anthropologie, une rupture « avec la vision de l'ethnologie classique et son parti pris d'équilibre, d'ancrage, de stabilité, avec l'interdépendance étroite milieuenvironnement-société » (Amiotte-Suchet, Floux, 2002 : 10).

Plus récemment, des anthropologues ont pu séjourner dans les camps pour y effectuer des recherches empiriques sur la condition de réfugié et celle du monde de l'humanitaire. Il s'est agi de donner la parole aux réfugiés, de les considérer comme des acteurs, afin de comprendre leur nouvelle identité et de restituer leurs stratégies. L'approche était résolument qualitative. Parmi les chercheurs anglo-saxons ayant travaillé dans des camps africains, citons Liisa Malkki (1995) et Julia Powles (2002) qui explorèrent les thèmes du monde social de l'exil et de l'identité réfugiée, la première, parmi les réfugiés hutu burundais en Tanzanie, la seconde, avec les réfugiés angolais en Zambie. Liisa Malkki recueillit les récits ancestraux de deux groupes de réfugiés hutu burundais, l'un rassemblé dans le site agricole de Mishamo, l'autre, dispersé 
dans la petite ville de Kigoma. Elle souligne les différences qu'elle observa entre ces deux groupes quant à la signification accordée à l'identité nationale, à l'histoire, et aux notions du chez soi et de l'exil : «Comparison of the camp and township settings revealed radical differences in the meanings that people ascribed to national identity and bistory, to notion of home and bomeland, and to exile as a collectively experienced condition» (Malkki, 1995: 2). Quant à Julia Powles qui résida dans le site de Meheba ouvert pour les réfugiés angolais en Zambie, elle s'attacha à montrer qu'il était déterminant de recueillir la parole des réfugiés, expression de leur histoire, de leur vie sociale et de leur culture : "Recording refugees' stories enables them to express their experiences and evaluate them within their own terms, historical, social and cultural $\gg^{5}$ (Powles, $2004: 20)$.

Si les anthropologues anglo-saxons ont été des précurseurs, il faut citer, parmi les anthropologues français, Michel Agier (2008), Marion Fresia (2009), Virginie Tallio (2006), Alice Corbet (2008) qui ont fourni des données de terrain précieuses sur les territorialités des espaces des camps, le fonctionnement et les paradoxes du monde de l'humanitaire, le changement social et la reconstruction de sociétés en exil.

Virginie Tallio (2007) a produit une ethnographie détaillée d'un espace humanitaire qui donne des clés pour comprendre la gestion d'un camp et le processus du rapatriement. Sa recherche s'inscrit dans les débats sur les liens entre identité et territoire. Alice Corbet (2008), quant à elle, met en lumière les évolutions sociales et culturelles d'une société en exil, la société sahraouie. Elle se penche plus particulièrement sur l'identité des jeunes nés dans les camps, une identité produite par les camps et par le paradoxe entre un monde idéel très prégnant et un monde réel.

${ }^{5}$ Recueillir les histoires des réfugiés leur permet d'exprimer leurs expériences et de les restituer avec leurs propres termes, historiques, sociaux et culturels.
Quant à Marion Fresia, elle livre un ouvrage capital, à la lumière d'une étude empirique minutieuse d'une expérience d'exil - celle des Mauritaniens réfugiés au Sénégal sur les processus de changements sociaux et identitaires induits par le déplacement forcé et par l'intervention humanitaire. Mettant en relief les normes et les catégories produites par le droit international des réfugiés et le droit international humanitaire, son étude permet de «mieux comprendre les effets concrets du transfert de ces normes et de ces catégories, d'origine occidentale, à des contextes sociopolitiques, historiques et culturels différents » (Fresia, 2009 : 347).

De plus, parmi les géographes de l'exil, citons les travaux de Kamel Doraï (2006 (a), (b)), qui étudie les dynamiques migratoires, «les questions du retour, de l'installation et du redéploiement spatial d'une diaspora de réfugiés ", celle des réfugiés palestiniens au Liban (Doraï, 2006 (a)). Il se penche sur les rapports entre réfugiés, acteurs politiques et dynamiques spatiales, à propos des réfugiés palestiniens dans les camps au Liban, des camps, "tissus de base de la société palestinienne dans l'exil», véritables " espaces de repli identitaire, social et économique » (Doraï, 2006 (b) : 94). Quant à Fabienne Le Houérou (2006, 2004), historienne et spécialiste des migrations forcées en Egypte et au Soudan ainsi qu'en Afrique orientale, elle explore la notion de "perte d'histoire» et s'intéresse aux territoires urbains produits par les réfugiés et au-delà, aux limites des concepts juridiques liés au monde des réfugiés à propos des réfugiés érythréens regroupés dans les camps soudanais.

Les sciences sociales se sont donc intéressées tardivement à cet espace d'étude, le camp, en y dénonçant tout d'abord le système d'aide. Le camp est alors appréhendé "comme une entité sociale à part entière, afin d'y observer les logiques de pouvoir et de contre-pouvoir déployées par les réfugiés et les institutions humanitaires » (Fresia, 2009 : 62). 
Alors que ces chercheurs ont produit des informations précieuses sur le monde des réfugiés et de l'humanitaire, à partir d'une approche des camps de réfugiés, la plupart d'entre eux ont été discrets sur leurs pratiques d'enquêtes et sur les difficultés rencontrées sur le terrain. À la lecture de leurs travaux, il est possible, toutefois, de regrouper leurs démarches selon trois types, l'observation participante, la comparaison et les passages multiples.

\section{Quelques méthodologies adoptées dans les camps de réfugiés}

«Immersion impossible », tels sont les termes utilisés par Pierre Centlivres lorsqu'il évoque les conditions de ses recherches dans les camps de réfugiés afghans au Pakistan : «la traditionnelle enquête de terrain n'est plus possible : participer, être l'un des leurs, planter sa tente...vous ne pouvez pas participer à la vie des réfugiés ». Impossible d'être l'un des leurs souligne Pierre Centlivres (Amiotte-Suchet, Floux, 2002: 12).

Pourtant, quelques jeunes anthropologues ont tenté d'adopter une approche d'observation participante. C'est le cas d'Alice Corbet qui a effectué un long travail de recherche dans les camps sahraoui en Algérie. Elle a choisi l'immersion dans la communauté réfugiée en résidant dans une famille, en se faisant en quelque sorte adopter par cette famille et en apprenant leur langue. C'est par cette intégration participante, souligne Alice Corbet, qui signifiait vivre avec les populations qu'elle a réussi à sortir de la victimisation, à dépasser cette notion, c'est-à-dire à voir ces personnes comme des acteurs (Corbet, 2008).

C'est aussi la démarche adoptée par Julia Powles qui vécut un an dans le site agricole de Meheba en Zambie et partagea la vie quotidienne d'une famille de réfugiés angolais dont elle a recueilli les récits de vie, les expériences vécues du déplacement forcé et la notion du chez soi qui donnèrent lieu à une thèse (Powles, 2000) et à une publication, parmi d'autres, centrée sur l'interprétation du récit biographique de Susanna Mwanna Uta, une réfugiée angolaise âgée (Powles, 2002). C'est encore le cas de Liisa Malkki (1995) qui a résidé plusieurs mois dans le site de Mishamo et dans la ville de Kigoma en Tanzanie.

D'autres chercheurs ont fait le choix non pas de résider dans un camp sur la longue durée mais de faire varier les échelles d'observation en diversifiant les cas d'étude. Marion Frésia (2009), par exemple, a étudié plusieurs camps de réfugiés mauritaniens dans la vallée du fleuve Sénégal, en y associant des villages jumelés, des regroupements spontanés, des quartiers urbains, éléments déterminants des dynamiques d'insertion. Elle est retournée dans chacun des lieux étudiés à plusieurs reprises au cours de séjours de quelques mois, en cherchant en quelque sorte à mener une véritable ethnographie compréhensive d'une configuration humanitaire. Toutefois, Marion Fresia comme Liisa Malkki expliquent qu'elles ont pu mener leurs recherches parce qu'elles étaient en situation de post-urgence, caractérisée à la fois par le désengagement des institutions humanitaires, et notamment du HCR, et par une faible médiatisation des réfugiés. Pour Marion Fresia, cette temporalité a présenté des avantages certains puisqu'elle avait libre accès aux camps de réfugiés et la possibilité de " pouvoir observer la vie des populations exilées en dehors des institutions qui les nomment et ainsi d'adopter un regard décalé sur son objet d'étude» (Fresia, 2009: 21). Quant à Liisa Malkki, elle a également travaillé en situation de post-urgence, 13 ans après l'arrivée des réfugiés hutu en Tanzanie. Son terrain débuta 2 mois après le hand-over du site agricole par le HCR au gouvernement tanzanien, une situation propice au recueil des histoires de vie de ceux qui étaient toujours présents (Malkki, 1995).

D'autres enfin ont eu l'opportunité de travailler par contrats avec des ONG ou le 
HCR. C'est le cas de Luc Cambrézy qui a participé à un bilan des dégradations environnementales liées à la présence des camps en dressant pour l'unité «environnement» du HCR des cartes de déforestation autour des camps de Dadaab au Kenya (Cambrézy, 2001(b)). C'est aussi le cas de Michel Agier qui s'est allié à l'ONG Médecins sans frontières, ce qui lui a permis de multiplier les missions de courte durée dans une diversité de situations de camps. Par cette alliance, le chercheur est obligé de devenir partie prenante de l'action humanitaire et de répondre à une demande de l'organisation, même s'il ne s'agit pas d'une commande. C'est «un terrain d'enquête inhabituel dans la recherche ethnologique » souligne Michel Agier (2008 : 104), dans la mesure où le chercheur «mène une recherche critique sur les camps, sans que cela passe jamais par une commande d'expertise opérationnelle qui aurait "cadré » à l'avance les réponses possibles » (Agier, 2008 : 104).

$\begin{gathered}\text { Observation participante sur une } \\ \text { missions courtes }\end{gathered}$
longue durée, ou avec des organisations
diachroniques, missions sur contrats avec le
HCR ou ces méthologies
humanitaires, toutes ces méthe àne
participent, chacune à leur façon, à une
connaissance approfondie du monde des
réfugiés et de l'humanitaire. La recherche
que j'ai menée à Ukwimi se situe dans une
posture méthodologique singulière car elle
s'inscrit dans le temps long par des passages
répétés et ne concerne qu'un site de réfugiés.

\section{Une enquête à passages répétés dans le site d'Ukwimi en Zambie}

Pour étudier la réalité de la politique d'asile zambienne et son adéquation avec la solution de l'installation sur place, j'ai mené une enquête à passages répétés dans le site agricole d'Ukwimi en Zambie. Ces recherches empiriques ont été conduites sous la forme de missions successives qui se sont déroulées sur une quinzaine d'années, de 1993 à 2007. J'ai choisi cette méthode d'approche parce qu'elle me permettait, par de courts séjours répétés, d'observer en temps réel les phases d'évolution du site agricole, tant au niveau démographique qu'économique et social, autant d'indices de compréhension de la politique d'asile zambienne.

Les enquêtes à passages répétés ou multiples sont des outils fréquemment utilisés par les démographes, les économistes, les professionnels et chercheurs de la santé dans les pays en développement en raison de l'absence d'un cadre statistique fiable. Elles sont généralement menées sous la forme d'enquêtes à la fois quantitatives et qualitatives, organisées le plus souvent à l'échelle nationale ou régionale pour évaluer par exemple le niveau de mortalité (exemple du Bénin étudié par Laourou, 1994) ou la croissance démographique (exemple d'Haïti étudié par Domenach, 1976). Elles sont aussi utilisées dans le cadre de grandes enquêtes sur les budgets-consommation, la nutrition ou encore les niveaux de vie, et sont effectuées à la demande d'organismes onusiens comme l'Unicef ou par des centres de recherche comme l'ORSTOM devenue l'IRD. Ce sont toujours des enquêtes extrêmement lourdes qui s'appuient sur un plan de sondage et un échantillonnage rigoureux, des passages successifs à intervalle régulier, des questionnaires détaillés et de nombreux enquêteurs afin de faire un suivi sur parfois plusieurs années d'un échantillon permanent de population.

Dans la mesure où j'étais seule pour mener ces enquêtes, où l'espace enquêté était réduit à quelques $\mathrm{km}^{2}$, où il était impossible de suivre un échantillon permanent de population, ma pratique d'enquêtes à passages répétés a été tout à fait particulière. Pourtant, pendant toutes ces années, j'ai tenté de conserver autant que possible les mêmes interlocuteurs. Le premier entretien était perçu en quelque sorte comme «le début d'une série d'entretiens et au-delà, d'une relation » (Olivier de Sardan, 1995). 
Toutefois, à chaque passage, certains de mes interlocuteurs étaient partis définitivement comme la plupart des réfugiés mozambicains lors du grand rapatriement de 1994. En revanche, d'autres groupes s'étaient installés à l'image des colons qui reprenaient les terres laissées vacantes par les réfugiés. Je devais perpétuellement renouveler mes interlocuteurs. En se basant sur mon échantillon initial d'une cinquantaine de personnes, seules une dizaine d'entre elles ont été des enquêtés permanents. Ils appartenaient à deux groupes, les autochtones villageois et les réfugiés mozambicains qui avaient refusé le rapatriement. Les rencontrer pendant une quinzaine d'années a permis de tisser des liens de confiance. À chaque rencontre, il était possible de revenir sur les questions posées précédemment et de les approfondir, de suivre les évolutions de leur situation en temps réel. La relation qui s'était instaurée au fil du temps autorisait - outre des entretiens libres - des discussions amicales autour de repas partagés ou à l'occasion de rencontres inopinées, toujours riches d'informations.

Ce n'était pas une enquête à passages renouvelés classique puisqu'il m'était impossible de suivre l'histoire de tous les groupes qui, à un moment ou à un autre, avaient été présents sur le site. C'était plutôt un terrain revisité sur le temps long qui permettait de s'imprégner de la réalité des évolutions, de créer des liens sans adopter l'observation participante. Ces enquêtes qualitatives ont été menées par des entretiens semi-directifs à chaque passage, auprès d'une cinquantaine de personnes issues des différents groupes formant la « communauté » d'Ukwimi, c'est-à-dire les autorités présentes sur le site (Refugee Officer, responsables d'ONG, chefs coutumiers, chefs de village, représentant du HCR), les réfugiés (Mozambicains, Angolais, Congolais, Burundais, Rwandais), les autochtones vivant dans et autour du site et enfin, les nouveaux colons. Ainsi, l'échantillonnage était le plus large possible puisqu'il s'agissait de prendre en compte le positionnement de tous les acteurs présents sur le site et interagissant autour de cette configuration humanitaire.

Cette méthode empirique ne pouvait s'appuyer que sur un échantillonnage aléatoire. Il m'était impossible de construire un échantillon représentatif sur le plan statistique puisque je ne disposais pas d'un cadre statistique fiable. En effet, les chiffres produits par les ONG et le HCR ne concernaient que les réfugiés, c'est-à-dire les personnes sous mandat. Les populations autochtones, présentes sur le site, étaient statistiquement invisibles. Face à cette difficulté, je choisis de me laisser guider par mon interprète qui était un autochtone, un pasteur pentecôtiste et aussi un instituteur. Ce triple statut lui conférait une large aura dans le site. Il est sûr qu'il me conduisit de préférence vers ses ouailles et vers les familles de ses élèves, mais ces personnes faisaient partie de tous les groupes présents sur le site.

De plus, j'attachais une grande importance à l'observation que j'associais à l'enquête par entretiens. J'ai toujours privilégié la rencontre des personnes enquêtées dans leur habitation ou dans leurs champs. L'entretien avait lieu avec le chef de ménage ou son épouse. Cette pratique m'obligeait à cheminer de village en village, à pied ou en bicyclette, permettant ainsi d'associer, aux entretiens, l'observation des lieux parcourus. Les retours sur le terrain étaient l'occasion de retrouver des paysages familiers dont on pouvait identifier les changements que je cherchais à expliquer. Ainsi, en parcourant le site agricole, il était facile de constater que ceux qui étaient mis à l'écart n'étaient pas les réfugiés mais les autochtones. C'était une géographie de parcours qui se faisait en marchant hors des sentiers battus des humanitaires. À la lumière de cette méthodologie empirique appliquée au cours de missions successives, j'interrogerai les difficultés méthodologiques et éthiques rencontrées pour produire mes 
propres données, que ce soit la phase de recueil des données, leur interprétation et leur restitution.

\section{Urgence, rapatriement, illégalité, trois temps forts de la vie du site agricole d'Ukwimi (Zambie)}

J'ai divisé mon approche selon trois grandes phases de la vie de ce site agricole que j'ai observées, période de l'urgence, période du rapatriement et période de l'illégalité. L'objectif de mes recherches était d'étudier la réalité du discours sur l'insertion et l'autosuffisance des réfugiés à travers l'étude de l'occupation de l'espace, des relations foncières et économiques entre réfugiés et autochtones.

\section{3, période de fin de l'urgence : accéder au terrain et légitimer sa présence}

J'ai découvert Ukwimi en 1992 en participant à la visite « organisée » de ce site agricole modèle du HCR, à l'issue d'un colloque qui venait de se tenir à Blantyre au Malawi sur le thème "First Country of Asylum and Development Aid in Malawi». Depuis 15 ans, la guerre civile dévastait le Mozambique et entraînait des exodes croissants vers les pays frontaliers dont le Malawi qui accueillait près d'un million de réfugiés dans de nombreux camps répartis dans tout le sud du pays.

\section{L'ONG Lutheran World Federation} $(\mathrm{LWF})^{6}$, principale ONG partenaire du HCR à Ukwimi, nous invita à parcourir le site, à découvrir les villages des réfugiés mozambicains (où nous sommes accueillis par des danses et des chants), le marché central (où nous sommes incités à acheter des produits artisanaux, du miel, etc.) et les multiples réalisations de la communauté

${ }^{6} \mathrm{La}$ Fédération mondiale Luthérienne est une ONG internationale basée à Genève et spécialisée dans le développement rural. Elle fut la principale partenaire du HCR dans les sites agricoles ouverts en Afrique orientale et australe. internationale (écoles, dispensaires, moulins à maïs, ateliers de formation divers, porcheries, poulaillers, etc.) de ce site façade, ce site vitrine du HCR. Nous étions en quelque sorte reçus comme des autorités officielles visitant un projet de développement rural que nous avions financé. Nous étions perçus comme faisant partie des donateurs du monde occidental. À l'issue de cette «visite guidée », je décide de revenir l'année suivante pour explorer l'autre face du miroir, c'est-à-dire la réalité de l'insertion de réfugiés hautement assistés dans un milieu local qui, lui, ne semble pas l'être.

L'objectif de ma recherche était d'évaluer le degré d'insertion des réfugiés en observant les relations foncières, économiques et sociales qu'ils avaient nouées avec la population locale. Malgré les liens que j'avais établis avec la LWF lors de ma première visite, je rencontrai de multiples difficultés pour accéder à nouveau au terrain, y mener des recherches et légitimer ma présence.

\section{Accéder au terrain}

Comme nous l'avons écrit dans une de nos précédentes publications collectives, «Il faudrait tout d'abord rappeler que l'on n'entre pas dans un camp comme dans un village choisi comme objet d'une monographie portant sur les systèmes agraires » (Lassailly-Jacob, Marchal, Quesnel, 1999 : 20). Lorsque j'ai voulu mener une recherche dans un site agricole géré par le HCR en Zambie, j'ai dû obtenir les autorisations de trois autorités en charge du lieu, le Commissariat zambien aux réfugiés dépendant du Ministère de l'intérieur, la délégation du HCR et la principale ONG partenaire du HCR, la LWF. Et pour obtenir ces autorisations qui demandèrent plusieurs jours d'attente à Lusaka, il me fallut présenter un projet de recherche détaillé afin de lever les suspicions. Comme le souligne Luc Cambrézy, «les chercheurs ne sont pas toujours les bienvenus dans le monde de 
l'assistance humanitaire. Au contraire des journalistes, alliés incontournables de ces organisations, les chercheurs inspirent souvent la méfiance. Celle-ci semble justifiée par une sorte d'intime conviction qu'il n'y aurait pas d'espace pour la recherche dans le contexte des populations réfugiées. » (Cambrézy, 2001 (a) : 23-24).

Accéder à un camp de réfugiés est donc conditionné à l'accord non seulement des autorités nationales mais aussi des institutions internationales qui $y$ interviennent. C'est un lieu sous surveillance, une enclave extra-territoriale dans laquelle le chercheur ne peut pénétrer qu'après contrôle de son identité et de ses intentions. À Ukwimi, l'entrée du site était fermée par une barrière surveillée en permanence par deux militaires zambiens qui contrôlaient votre identité et vos autorisations. Ces vérifications, comme le remarque Michel Agier, symbolisent « le passage non pas dans une prison mais dans un autre régime de gouvernement et de droits» (Agier, 2008 : 111). Arrivée dans le site, je dus me présenter au Refugee Officer zambien, au représentant du HCR et au représentant de LWF. Je devenais à la fois hôte du pays d'accueil et de la communauté internationale mais je n'étais plus sous la tutelle de la chefferie traditionnelle. Le chef coutumier, première personne à saluer dans un contexte de ruralité, avait perdu toute prééminence. Il était évincé de son statut par la confiscation d'une partie de son territoire par le HCR et par l'État zambien.

Ces difficultés d'accès sont aussi mentionnées par d'autres chercheurs comme Virginie Tallio (2012) pour entrer dans le camp de Nkondo en RDC ou Alice Corbet qui dut obtenir une lettre du Front Polisario pour pouvoir se rendre dans les camps sahraoui en Algérie. Celle-ci souligne que l'accès aux camps a demandé une longue préparation dans la mesure où elle était doctorante et non pas membre d'une organisation humanitaire ou journaliste (Corbet, 2008). Liisa Malkki parle aussi des difficultés qu'elle eut pour obtenir non seulement une autorisation de recherche nationale, mais aussi une autorisation spéciale délivrée par le ministère de l'Intérieur pour entrer dans le camp de Mishamo en Tanzanie. Elle souligne que ces difficultés administratives pour obtenir un droit d'accès et de séjour dans un camp de réfugiés sont significatives des enjeux politiques cruciaux que représentent la question des réfugiés: "Since "refugees》 is a category often seen as politically sensitive - even an issue of national security - obtaining research permission and doing research in this area can be an unusually complex process. » (Malkki, 1995 : 47). Et ces difficultés administratives se doublent généralement d'un problème d'accessibilité. En effet, les camps ou les sites agricoles sont souvent isolés.

\section{Atteindre le site}

Comme de nombreux sites de réfugiés, celui d'Ukwimi était localisé à l'écart. Pour atteindre le site, implanté à $470 \mathrm{~km}$ de Lusaka dans la province de l'Est et à une centaine de $\mathrm{km}$ de la frontière mozambicaine, il fallait quitter, à la hauteur de la petite ville de Petauke, la route principale, asphaltée, qui relie Lusaka à Lilongwe, puis emprunter vers le nord une piste sinueuse et dégradée de $70 \mathrm{~km}$. Si, en saison sèche, le parcours des $70 \mathrm{~km}$ durait 3 à 4 heures, en saison des pluies, en revanche, le site pouvait être inaccessible pendant plusieurs jours ${ }^{7}$. Cette mise à l'écart se retrouve dans de nombreuses situations comme au Kenya où les camps de Dadaab qui abritent les réfugiés somaliens sont situés dans une zone isolée, quasi désertique et difficilement accessible (Cambrézy, 2001 (a)). Liisa Malkki évoque aussi les difficultés d'accès au site de Mishamo.

\footnotetext{
7Pendant toute la période de distribution de l'aide internationale, la piste fut entretenue par des fonds internationaux. La demande de renouvellement de l'aide internationale a toujours associé les travaux d'entretien de cette piste vitale pour le développement du site agricole.
} 
Camps de réfugiés et sites agricoles sont souvent isolés, dans des espaces enclavés, mal desservis. Luc Cambrézy considère le camp comme un moyen d'endiguement des déplacements des réfugiés, c'est-à-dire " le plus sûr moyen de retenir les réfugiés dans un certain espace qui, sans être nécessairement clos, est localisé et géré de façon à limiter les mouvements de population » (Cambrézy, 2001 (a) : 82). Difficilement accessibles, ils interrogent la faisabilité des objectifs de l'aide à l'installation sur place, à savoir l'autonomie et l'intégration locale. Comment les réfugiés peuvent-ils devenir autosuffisants et s'intégrer dans ces lieux à l'écart?

Si les sites de réfugiés sont peu accessibles administrativement et géographiquement, il existe aussi d'autres situations d'accès et de travail auprès de réfugiés tout aussi difficiles. Par exemple, lorsque le HCR passa commande à l'Institut des études africaines de l'université de Zambie d'un survey dans la province du Nord-Ouest de la Zambie en 1982 pour mieux connaittre la situation des réfugiés angolais, zaïrois et namibiens auto-installés dans les régions frontalières, les chercheurs rencontrèrent de multiples difficultés pour accéder à leur terrain et pour y travailler. Comment recenser une population invisible qui se cachait et était cachée par la population locale ? (Freund et Kalumba, 1986).

\section{Travailler sous tutelle}

Le représentant de l'ONG LWF m'avait donné une autorisation de séjour, me logeait dans la guest house du HCR et me véhiculait parfois. J'avais une table de travail dans l'un de leurs bureaux et j'avais accès à tous leurs rapports et statistiques. J'étais en quelque sorte leur "expert», je travaillais avec leurs bénéficiaires, les réfugiés, sur leur territoire, un espace qu'ils avaient remodelé de leur empreinte administrative ${ }^{8}$ et marqué de leur vocabulaire? En échange, je m'engageais à leur fournir mes rapports de mission et une cartographie des lieux parcourus, des cartes que je retrouvais épinglées dans leurs bureaux et ceux des ONG œuvrant sur le site. J'étais ainsi dans une logique de don/contre-don.

Face à cette présence institutionnelle forte, il m'était impossible de mener une recherche idéale, basée sur une relation duale, enquêteur-enquêté avec un informateur privilégié, l'interprète. Je devais répondre aux demandes de l'organisation en contrepartie des moyens de travail qu'ils m'offraient. Cette situation est également soulignée par Pierre Centlivres dans un entretien à propos des recherches qu'il menait dans les camps afghans au Pakistan : «Vos partenaires sont différents de ceux de la classique étude de communauté. Vos interlocuteurs sont des réfugiés bien sûr, mais c'est aussi l'ensemble du personnel de gestion des camps, les volontaires des ONG, les experts des Organisations Internationales (OI). Un échantillon de la mondialisation! Ces gens font également partie du jeu, n'estce pas?» (Amiotte-Suchet, Floux, 2002: 12).

Toutefois, je refusais de n'étudier que les seules interactions entre réfugiés et humanitaires. J'élargissais mon champ d'enquête à l'ensemble des groupes vivant sur le site et à leurs interactions. Je souhaitais sortir de l'espace connu, l'espace «vitrine» des institutions internationales et des ONG dont les représentants sillonnaient cette zone du site à bord de leurs véhicules ou de leurs motos. Cet espace avait été conçu pour faciliter le déploiement du dispositif humanitaire. Les cases des réfugiés étaient alignées à intervalle régulier en villages-rues

${ }^{8}$ Le territoire avait été divisé en deux zones administratives, Ukwimi A et Ukwimi B, ayant chacune leurs beadquarters. Chacune des zones administratives était divisée en 2 ou 3 satellites.

${ }^{9}$ Les villages étaient numérotés. Les centres administratifs avaient reçu les noms de certains villages autochtones. 
le long des pistes et le personnel d'encadrement et les membres des ONG, étaient rassemblés dans les maisons en dur des quartiers administratifs situés à proximité de l'entrée du site pour des raisons de sécurité.

C'était un espace uniformément attribué $^{10}$, surveillé et contrôlé de près. Les occupants, c'est-à-dire les réfugiés, devaient se plier à des directives strictes, faisaient l'objet de recensements répétés et de visites régulières de la part du personnel d'encadrement. C'était l'espace visible, la devanture que le visiteur, c'est-à-dire le donateur, était invité à voir et à apprécier. Mon intérêt se portait aussi sur l'autre type d'espace qui était à l'écart, situé le long des sentiers, à proximité des marigots et sur les marges du site. C'était l'espace des autochtones, ignoré des autorités car il était hors de leur contrôle. C'était une communauté fantôme dont la présence était tolérée mais peu connue et non recensée.

\section{Enquêter en période d'urgence}

Pendant cette première mission de recherche, je côtoyais de nombreuses ONG qui intervenaient dans de multiples domaines (santé, distribution alimentaire, formation agricole, etc.). Le chercheur a-t-il sa place dans un tel contexte? Y-a-t-il un espace pour la recherche dans la période de l'urgence?

J'occupais une place à part dans le sens où je n'étais pas confrontée à des priorités opérationnelles. Je n'étais ni dans l'action, ni dans le témoignage d'évènements immédiats. Mon rôle était d'observer les multiples interactions entre les ONG et les réfugiés, entre les réfugiés et les autochtones, entre les autochtones et les colons et de transmettre au HCR et aux ONG les résultats de mes observations, quitte à dénoncer des situations intolérables.

${ }^{10}$ Chaque famille réfugiée recevait un lot de $600 \mathrm{~m}^{2}$ pour bâtir son habitation ainsi qu'une parcelle de terre cultivable de 2 ha.
En 1993, certains réfugiés étaient présents depuis 6 ans tandis que d'autres continuaient à arriver. L'aide internationale était encore très présente. Lors de mes enquêtes dans les villages des réfugiés mozambicains, les chefs de famille rencontrés me présentaient dès le début de l'entretien leur carte de réfugié sans aucune demande de ma part. C'était une habitude qu'ils avaient prise lors des nombreux entretiens auxquels ils étaient soumis par les humanitaires. J'essayai de les faire parler de leur passé et de leur futur, mais j'étais toujours ramenée aux préoccupations du présent. Ils me répétaient : Nous avons besoin de cowvertures, de sel, d'buile. Nous ne trouvons pas de travail à la tâche. Les Zambiens ici sont trop pauvres. Ils délivraient un discours fait de plaintes. Cet aspect de la recherche en période d'urgence est aussi évoqué par Pierre Centlivres à propos de ses enquêtes dans les camps afghans, "Les gens ne se soucient guère de vous raconter leurs vieilles coutumes. Ce qu'ils veulent, c'est que vous les aidiez, que vous obteniez une machine à coudre pour les veuves, une ration alimentaire pour les enfants, une inscription sur les listes du HCR pour recevoir des secours. Donc là, réellement, tout ce qu'on a appris de l'enquête de terrain ne sert pas à grand chose; l'anthropologie classique est remise en question » (Amiotte-Suchet, Floux, $2002:$ 12).

Le recueil de ce discours pose la question de la représentation que des populations réfugiées se font du chercheur indépendant. Les propos recueillis révélaient qu'ils me voyaient comme appartenant au monde des humanitaires. Pourtant, ma façon de travailler avec eux était différente. Je ne les convoquais pas à des réunions, je n'imposais rien, je les rencontrais dans leur case ou dans leurs champs, posant seulement des questions et les écoutant. Pour les réfugiés, toute personne venant les enquêter ne pouvait qu'appartenir au monde des humanitaires d'autant plus qu'elle était blanche, qu'elle résidait dans la guest house 
construite par le HCR et qu'elle utilisait parfois les véhicules de LWF.

J'ai senti cette même perception lorsque je suis retournée mener des enquêtes dans les zones de rapatriement des réfugiés au Mozambique (Lassailly-Jacob, 2010). Il fut très difficile de travailler dans une zone où le HCR et de nombreuses ONG avaient fait de multiples surveys les années précédentes. Toutes les personnes rencontrées pensaient que nous appartenions à l'ONU, même si ce n'était pas inscrit sur notre véhicule, et elles s'attendaient à être rémunérées pour leur entretien comme elles l'avaient été autrefois. Comprenant alors que ce temps était révolu, elles se sont soumises de mauvaise grâce aux enquêtes en disant qu'elles n'étaient plus des rapatriés et qu'elles étaient fatiguées d'être interrogées. La présence de l'ONU et celle des ONG avaient biaisé la relation d'enquête.

Cette situation a également été observée par d'autres chercheurs comme Julie Beaujard, ethnologue, qui a mené des enquêtes auprès des réfugiés d'Afghanistan et du Myanmar accueillis à New Delhi et sous protection des Nations Unies et qui souligne : "Généralement, le chercheur est perçu par les réfugiés comme appartenant à l'organisation qui intervient auprès d'eux et est considéré comme l'un de ses employés » (Baujard, 2005: 129). Elle était « ou bien crainte et évitée, ou bien "utilisée » comme porte-parole de leurs revendications et de leurs plaintes »(Baujard, 2005 : 131). Liisa Malkki, elle aussi, mentionne qu'elle s'était présentée comme un chercheur indépendant, mais les réfugiés ne comprenaient pas ce qu'elle venait faire et pourquoi elle les interrogeait sur leur histoire. La suspicion s'était très vite installée. Les premiers temps de son enquête, elle fut considérée comme une espionne envoyée par le gouvernement burundais et reçut le surnom de «femme Tutsi» parce qu'elle était grande et mince comme une Tutsi (Malkki, 1995).
Malgré ces difficultés, je me suis attachée à identifier les éléments qui jouaient en faveur ou à l'encontre de l'insertion des réfugiés dans le milieu local. Enquêtes et observations ont permis de découvrir que les tensions qui freinaient l'insertion des réfugiés étaient surtout dues à l'inégalité engendrée par la distribution de l'aide humanitaire dans un contexte régional de grande pauvreté. Les organismes d'assistance jouaient un rôle primordial dans la qualité des relations de voisinage entretenues entre autochtones et réfugiés.

En 1993, six ans après son ouverture, le site n'était toujours pas déclaré autosuffisant. L'autonomie des réfugiés n'avait jamais été réalisée et leur intégration était freinée par l'isolement du site et les inégalités engendrées par la distribution de l'aide humanitaire. Le HCR était toujours présent et l'aide internationale continuait d'être distribuée. De nouveaux réfugiés arrivaient encore pendant qu'un plan de rapatriement se mettait en place. La solution de l'aide à l'installation sur place n'était réalisée que par les infrastructures mises en place dans le site.

\section{5-1999, \\ période de fin du rapatriement : perdre ses interlocuteurs, en trouver d'autres et travailler sous une nouvelle juridiction}

De retour à Ukwimi en 1997, je constate que la majorité des 25000 réfugiés mozambicains ont quitté le site au cours d'un vaste rapatriement organisé par le $\mathrm{HCR}^{11}$ en 1994. Suite aux accords de paix signés à Rome le 4 octobre 1992 entre le gouvernement du Mozambique et la Resistência Nacional Moçambicana (RENAMO), la clause de cessation du statut de réfugié est appliquée en 1995. Cette même année, le HCR se retire du site et l'aide internationale s'arrête. Le site est déclaré «fermé » en tant que camp de réfugiés sous administration onusienne. Il n'a jamais été reconnu comme

\footnotetext{
${ }^{11} \mathrm{En}$ Zambie, de juillet à décembre 1994, de nombreux bus furent affrétés par le HCR pour rapatrier les réfugiés d'Ukwimi.
} 
auto-suffisant et localement intégré comme le stipulait le HCR.

Ukwimi Mozambican Refugee Settlement change de nom et d'identité administrative. Il devient périmètre agricole gouvernemental et prend le nom d'Ukwimi Resettlement Scheme en passant sous la juridiction du Département de la Réinstallation sous l'autorité du bureau de la vice-présidence.

Le territoire du site change de juridiction et est brutalement remodelé. Les terres abandonnées par les réfugiés sont cadastrées et mises à la disposition de colons zambiens. Enfin, un petit groupe de Mozambicains refuse le rapatriement et décide de rester malgré une situation d'illégalité. Ces transformations brutales et multiples se traduisent par la disparition d'un grand nombre de mes enquêtés, par l'élargissement de mon échantillon et par de nouvelles questions de recherche. Le rapatriement a-t-il été librement consenti ? N'a-t-il pas été anticipé ? Que signifiait être réfugié en Zambie? Répondre à ces interrogations apporta un éclairage sur la signification que le gouvernement zambien donnait au statut de réfugié : des hôtes temporaires invités à mettre leur force de travail au service du pays d'accueil tant que la communauté internationale les prend en charge (Lassailly-Jacob, 2003). La solution de l'aide à l'installation sur place n'avait durée que sept ans.

\section{Reconstruire un échantillon}

En 1997, cheminant à la recherche des premiers enquêtés Mozambicains, je découvre des villages fantômes où le silence est impressionnant. Dans certains villages, quelques cases abandonnées par leurs premiers occupants sont occupées par des nouveaux venus, des colons de la province de l'Est. Les terres sont en cours de cadastrage en parcelles de 5 ha et suscitent de fortes tensions entre les nouvelles autorités et les autochtones qui perdent leur patrimoine foncier. Quelques réfugiés sont restés.

Travailler dans un camp de réfugiés implique le risque de voir son terrain se fermer brutalement. C'est un terrain incertain qui se fait, se défait et se refait très rapidement. Je dus reconstruire un échantillon en intégrant aux autochtones et à quelques ex-réfugiés restés sur le site, les nouveaux venus, c'est-à-dire les colons zambiens venus prendre la place des réfugiés. Les deux groupes permanents que j'ai pu suivre pendant toute la durée de mon enquête ont été les autochtones et ces quelques réfugiés ayant refusé le rapatriement et de ce fait, étant devenus illégaux.

\section{Travailler sous une nouvelle juridiction}

Le HCR s'était retiré en 1995, c'était la fin de l'aide internationale mais son ONG partenaire, le LWF, avait été reconduit pour superviser le site et y poursuivre ses actions de développement agricole. Cette ONG luthérienne était associée à 3 autres ONG confessionnelles, le Christian Council of Zambia, le Catholic diocese of Chipata et les Young Men Christian Association. Les églises se multipliaient sur le site.

De plus, j'étais désormais dans un périmètre agricole étatique où les colons affluaient. Les terres mises en valeur par les réfugiés n'avaient pas été rendues aux autochtones comme cela avait été promis par les autorités. Elles devenaient propriété de l'État et étaient cadastrées pour être ensuite distribuées avec un titre foncier.

Enfin, 31 familles mozambicaines, soit quelque 143 personnes, avaient refusé de quitter le site et avaient été regroupées dans d'anciens villages. Trois communautés se côtoyaient : les colons, les autochtones et les ex-réfugiés mozambicains, et les tensions foncières étaient palpables. 


\section{0-2007, enquêter sur l'urgence et sur l'illégalité, restituer et témoigner}

En 1999, le site agricole est toujours en cours de transformation en un périmètre étatique; le cadastrage des terres se poursuit malgré les résistances des populations autochtones. Les colons zambiens continuent d'affluer et les ex-réfugiés mozambicains sont candidats pour acquérir des propriétés de 5 ha, une demande impossible à réaliser du fait de leur situation d'illégalité. L'année suivante, la situation se complexifie à nouveau avec la création d'un nouveau camp du HCR dans une partie du site. Il s'agit alors d'enquêter sur de nouvelles situations d'urgence et d'illégalité.

\section{Enquêter sur de nouvelles situations d'urgence}

En décembre 2000, le gouvernement, en accord avec le HCR, ouvre un nouveau camp de réfugiés dans la partie nord-ouest du site. Ukwimi redevient un camp du HCR. La barrière à l'entrée du site réapparaît, un nouveau Refugee Officer s'installe, une caserne de militaires s'établit à l'entrée du camp. Il s'agissait de faire venir des familles d'anciens combattants angolais du parti de l'União para a Independência Total de Angola (U.N.I.T.A.), blessés pour la plupart, qui résidaient dans un autre site agricole, celui de Meheba. Le gouvernement voulait séparer ces anciens combattants des civils angolais résidant sur ce site. Quelques mois plus tard, 200 jeunes burundais et rwandais demandeurs d'asile, ayant transité par la RDC, étaient amenés dans ce nouveau camp à Ukwimi depuis les prisons de Manza et de Kasama situées à proximité de la RDC. Dans ce nouveau camp de réfugiés transformé en site agricole, l'aide internationale était à nouveau dispensée et de nouveaux bâtiments (école, dispensaire, commissariat) étaient construits.

Mener des enquêtes auprès de ces jeunes ou auprès des soldats démobilisés qui venaient d'arriver était particulièrement difficile. Il me fut impossible d'enquêter sans la présence de deux militaires à mes côtés. De plus, ces réfugiés nouvellement arrivés étaient traumatisés et souffrants. Ils m'assimilaient bien sûr au HCR et délivraient un discours formaté de victime. À nouveau, j'étais utilisée comme porteparole de leurs revendications et de leurs plaintes.

\section{Enquêter sur des situations d'illégalité}

En 1999 puis en 2007, j’ai travaillé avec les ex-réfugiés mozambicains qui avaient refusé le rapatriement et dont la présence était toujours tolérée à Ukwimi. Par leurs conditions de vie, ils étaient devenus des présents-absents, poussés subrepticement à rentrer chez eux. Des " présents » parce qu'ils étaient considérés et traités comme des colons au même titre que les autres, mais des absents car en refusant le rapatriement, ils avaient perdu leur statut de réfugiés et étaient devenus des étrangers en situation illégale. Ils s'étaient vus retirer leur statut par le HCR, devenant de ce fait hors la loi dans le pays d'accueil. Dépourvus de papiers d'identité, ils étaient soumis à de multiples restrictions qui faisaient d'eux des exclus. Leur principale revendication était d'obtenir une carte nationale d'identité, une regie de couleur bleue (National Registration Card).

Ces réfugiés étaient devenus des sanspapiers parce que la politique d'asile nationale représentée par le Refugee Control Act était déconnectée de la politique d'immigration dans laquelle elle était supposée s'insérer. Ainsi, cette politique d'asile ne donnait aucune chance aux réfugiés d'accéder s'ils le souhaitaient au statut de migrant économique et encore moins à celui de citoyen. Il était impossible pour un ex-réfugié d'obtenir la naturalisation.

C'était donc une communauté en attente non pas d'un retour mais d'une impossible légalisation matérialisée par une carte d'identité. Comme le soulignait l'un des 
ex-réfugiés mozambicains "Having a "regie" means stop being called a refugee because it's an insult and it means being different. We want to be one of them. We want to be free and belong to this place. We want to stop being visitors without rights. »

\section{Restituer les données : une question éthique}

Les enfants des ex-réfugiés mozambicains avec lesquels je travaillais depuis des années étaient devenus adultes. Scolarisés en Zambie, ils cherchaient du travail mais sans carte d'identité, ils ne pouvaient fonder leur propre entreprise ou se faire embaucher. Leur seule façon d'obtenir une carte d'identité était de s'en procurer une frauduleusement comme cela est assez fréquent en Zambie. Mais, pour se procurer cette carte, il leur était impossible de rester dans le site où ils étaient stigmatisés comme réfugiés. Ils auraient été aussitôt dénoncés, arrêtés et emprisonnés. Leur seule issue était de devenir invisible en ville pour obtenir frauduleusement cette green identity card qui leur permettait de trouver du travail en Zambie. Ils étaient donc partis vivre dans les villes proches, une information qui m'avait été transmise par leurs parents. Pour des raisons éthiques, il m'était impossible de restituer les résultats de mes enquêtes concernant les destinations de ces jeunes sans les mettre en danger. Impossible également de dévoiler les itinéraires adoptés par les ex-réfugiés pour aller faire du commerce au Mozambique ni de parler des marchandises qu'ils rapportaient. Peut-on publier des résultats d'enquêtes qui peuvent porter préjudice aux populations enquêtées? Comme le soulignent Florence Bouillon, Marion Fresia et Virginie Tallio dans l'introduction de leur ouvrage "Terrains sensibles. Expériences actuelles de l'anthropologie » : «Portant sur des thèmes sensibles politiquement ou socialement, sur des pratiques dissimulées, ou encore sur des vécus construits sur fond de souffrance, la divulgation de résultats d'enquêtes comporte certains risques pour les groupes étudiés.
Elle peut aussi venir rompre la relation de confiance et de confidentialité établie avec ses enquêtés. En définitive, que doit-on dire ou ne pas dire? Pour qui ? Et comment?» (Bouillon, Fresia, Tallio, 2005 : 26).

Témoigner: jusqu'où le chercheur peut-il s'engager?

J'ai eu à témoigner d'une situation insoutenable, celle de 2001 lorsque le nouveau camp venait de s'ouvrir, accueillant des soldats blessés de l'UNITA et leurs familles. Dans ce nouveau camp, établi à la hâte, seuls deux infirmiers étaient présents et les rations alimentaires n'arrivaient qu'épisodiquement. Les réfugiés souffraient de famine, manquaient de soins et étaient oubliés du siège du HCR à Lusaka. De retour à Lusaka, je dénonce cette situation sur plusieurs radios locales...et je risque l'expulsion ${ }^{12}$. S'il est impossible de ne pas s'engager face à une telle situation, jusqu'où le chercheur peut-il s'impliquer sans se mettre en danger?

\section{Conclusion}

Il est sûr que toute recherche en sciences sociales présente des difficultés méthodologiques. Comme le souligne JeanPierre Dozon, "Les terrains ethnologiques ont toujours été des terrains sensibles. Comment auraient-ils pu ne pas l'être dès lors que la discipline a voulu se fonder sur la confrontation effective avec les us et coutumes des autres et ne faire science, par l'induction, la comparaison, la théorisation, qu'en s'éprouvant d'abord et avant tout comme expérience d'immersion dans les univers culturels les plus variés ainsi qu'entendait l'exprimer la formule canonique d'observation participante » (Dozon, 2005 : 7).

Mais certains terrains sont plus sensibles que d'autres. C'est le cas d'un camp

\footnotetext{
${ }^{12}$ Toutefois, dans les jours suivants, une délégation du HCR s'est enfin rendue à Ukwimi pour examiner la situation et prendre des mesures.
} 
de réfugiés en raison de ses fortes implications politiques et des dynamiques accélérées qu'il connaît. Un camp est brutalement remodelé par des entrées et des départs soudains, des successions de populations et de juridictions. Et ces évolutions rapides sont le reflet d'une politique d'asile qui, ici, montre bien que la solution de "l'aide à l'installation sur place » s'achève dès la déclaration du handed-over, c'est-à-dire le retrait du HCR et de l'aide internationale. Les réfugiés accèdent au territoire zambien à condition de le mettre en valeur mais cet accès est contrôlé dans le temps et dans l'espace. La politique d'asile zambienne tire partie de la présence des réfugiés et de leur force de travail pour bénéficier des investissements de la communauté internationale, une façon d'aménager son territoire et de mieux le contrôler.

Dans une telle configuration, le chercheur doit «bricoler » pour enquêter sur des situations d'urgence ou d'illégalité. Aussi, cette méthode d'enquêtes à passages répétés n'a-t'elle pu être conforme au modèle classique, mais elle a eu l'avantage de mettre en lumière les temps forts de la vie d'un site de réfugiés, apportant ainsi des informations précieuses sur l'illusion de «l'aide à l'installation sur place ».

Véronique Lassailly-Jacob Professeur de géographie Université de Poitiers/CNRS Migrinter - UMR 7301 vlassail@univ-poitiers.fr

\section{Bibliographie}

Agier, Michel (2008) Gérer les indésirables: des camps de réfugiés au gouvernement bumanitaire, Paris, Flammarion, 352 p.

Amiotte-Suchet, Laurent; Floux, Pierre (2002) Voyage avec Pierre Centlivres, de l'Afghanistan aux communautés transnationales, ethnographiques.org, $\mathrm{n}^{\circ} 1$, [en ligne, réf. du 05/02/2012] URL : $<$ http://www.ethnographiques.org/2002/C entlivres, Amiotte-Suchet,Floux.html>

Baujard, Julie (2008) Identité "réfugié ", identité transversale: les réfugiés à Delhi au sein des dynamiques institutionnelles, communautaires et associatives, Marseille, Université AixMarseille I, 555 p. Th. Doct : Anthropologie : Marseille : 2008.

Baujard, Julie (2005) Ni espionne, ni avocate: la place ambiguë de l'ethnologue auprès des réfugiés (HCR, New Delhi, Afghanistan et Birmanie) in Bouillon, Florence; Fresia, Marion et Tallio, Virginie (eds), Terrains sensibles: expériences actuelles de l'anthropologie, Paris, Édition de l'EHESS, pp. 125-143 (Dossiers Africains).

Begbeider, Yves (1999) Le Haut Commissariat des Nations Unies pour les réfugiés, Paris, PUF, 127 p. (Que sais-je ? n³489).

Bouillon, Florence; Fresia, Marion; Tallio Virginie (eds) (2005) Terrains sensibles: expériences actuelles de l'anthropologie, Paris, Édition de l'EHESS, Centre d'Etudes Africaines, 208 p. (Dossiers Africains).

Cambrézy, Luc (2001(a)) Réfugiés et exilés, crise des sociétés, crise des territoires, Paris, éditions des archives contemporaines, $216 \mathrm{p}$.

Cambrézy, Luc (2001(b)) Camps de réfugiés et environnement au Kenya: enjeux et contradictions, in Cambrézy, Luc ; LassaillyJacob, Véronique (eds) Populations réfugiées : de l'exil au retour, Paris, éditions de l'IRD, pp. 211-232 (Colloques et Séminaires). 
Corbet, Alice (2008) Nés dans les camps: changements identitaires de la nouvelle génération de réfugiés sabraouis et transformation des camps, Paris, Édition de l'EHESS, 398 p. Th. doct : Anthropologie. : Paris : 2008.

Domenach, Hervé (1976) Problématique et méthodologie de l'enquête à passages répétés en Haït, 1971-1975, Cabiers ORSTOM, Série Sciences Humaines, Vol XIII, n³, pp. 243-256.

Doraï, Mohamed Kamel (2006 (a)) Les réfugiés palestiniens du Liban, une géographie de l'exil, Paris, CNRS éditions, 252 p.

Doraï, Mohamed Kamel (2006 (b)) Le camp de réfugiés palestiniens d'Al Buss à Tyr: ségrégation et précarité d'une installation durable, Bulletin de l'Association de Géographes Français, n¹, pp. 93-104.

Dozon, Jean-Pierre (2005) Préface in Bouillon, Florence ; Fresia, Marion ; Tallio, Virginie (eds) Terrains sensibles: expériences actuelles de l'anthropologie, Paris, Édition de l'EHESS, Centre d'Etudes Africaines, pp. 711 (Dossiers Africains).

Fresia, Marion (2009) Les Mauritaniens réfugiés au Sénégal: une anthropologie critique de l'asile et de l'aide bumanitaire, Paris, l'Harmattan, 382 p.

Freund, Paul J.; Kalumba, Katele (1986) Spontaneously Settled Refugees in Northwestern Province, Zambia, International Migration Review, Vol. XX, N¹, pp. 299-312.

Harrell-Bond, Barbara E. (1986) Imposing Aid: emergency assistance to refugees, Oxford, Oxford University Press, 440 p.

Laourou, Martin H. (1994) Estimation de la mortalité au Bénin à partir d'une enquête à passages répétés, Population, Vol. 49, Nº1, pp. 119-143.

Lassailly-Jacob, Véronique; Marchal, JeanYves; Quesnel, André (eds) (1999) Déplacés et réfugiés: la mobilité sous contrainte, Paris, IRD, 504 p. (Colloques et séminaires).

Lassailly-Jacob, Véronique (2010) Forced migration in Africa: a new but overlooked category of refugees, in Audebert, C. \& Dorai, K. (eds) Migration in a Globalized World: New Research Issues and Prospects, Amsterdam, Amsterdam University Press, pp. 191-202.
Lassailly-Jacob, Véronique (2003) Conditions d'accueil et insertion des réfugiés dans un pays d'Afrique australe : la Zambie, in Guillon, M. ; Legoux, L.; Ma Mung, E. (eds) L'asile politique entre deux chaises: Droits de l'Homme et gestion des flux migratoires, Paris, l'Harmattan, pp. 245-265.

Le Houérou, Fabienne (2004) Migrants forcés éthiopiens et érythréens en Egypte et au Soudan: passagers d'un monde à l'autre, Paris, l'Harmattan, 201 p.

Le Houérou, Fabienne (2006) Les territoires usurpés des ex-réfugiés érythréens dans le nord-est soudanais, Bulletin de l'Association de Géographes Français, n 1 , pp. 62-75.

Malkki Liisa H., (1995) Purity and Exile. Violence, Memory, and National Cosmology among Hutu refugees in Tanzania, Chicago \& London, The University of Chicago Press, 352 p.

Olivier de Sardan, Jean-Pierre (1995) La politique du terrain, Enquête, Les terrains de l'enquête, [en ligne, réf. du 26/04/2012] URL :

$<\underline{\text { http://enquete.revues.org/document263.h }}$ $\underline{\mathrm{tml}}>$

Powles, Julia (2000) Road 65: a narrative ethnography of a refugee settlement in Zambia, Oxford, University of Oxford, $746 \mathrm{p}$. (Thesis).

Powles, Julia (2002) Home and Homelessness : The Life History of Susanna Mwana-Uta, an Angolan Refugee, Journal of Refugee Studies, Vol. 15, n¹, pp. 1-101.

Powles, Julia (2004) Life history and personal narrative: theoretical and methodological issues relevant to research and evaluation in refugee contexts, New issues in refugee research, UNHCR, n¹06, 24 p.

Tallio, Virginie (2006) L'humanitaire comme producteur de territoire : l'exemple du camp de Nkondo ouvert aux réfugiés angolais en R.D.C., Bulletin de l'Association de Géographes Français, n¹, pp. 39-49. 
Tallio, Virginie (2007) La fabrique du réfugié: du camp au rapatriement, lieux et processus de la construction $d u$ "réfugié ». L'exemple des camps de réfugiés de Dadaab (Kenya) et de Nkondo (RDC), Paris, Édition de l'EHESS, 343 p. Th. Doct. : Ethnologie : Paris : 2007.

Tallio, Virginie (2012) La construction de la catégorie "réfugiée» dans un camp en R.D.C. : rôle de l'institution, stratégies des exilés et posture du chercheur, e.migrinter, $\mathrm{n}^{\circ} 9$, pp. 18-28.
U.S. Committee for Refugees (1993) World Refugee Survey-1993, Washington D.C., 160 p. (Country Reports). 